\title{
Desencuentros con la Formación Docente para la Justicia Social. Currículos y Actitudes sobre Democracia y Ciudadanía
}

\author{
Disagreements with Teacher Education for Social Justice. \\ Curriculums and Attitudes about Democracy and Citizenship
}

\author{
Desencontros com a Formação Docente para a Justiça Social. \\ Currículos e Atitudes sobre Democracia e Cidadania
}

Ricardo Cuenca *
Sandra Carrillo
Luciana Reátegui
Instituto de Estudios Peruanos

\begin{abstract}
Con el regreso de la democracia en el 2001, el interés del sistema educativo peruano por la formación ciudadana y la democracia se vio reflejado en la incorporación de estos temas en los diseños curriculares de la formación básica, en la gestión de las escuelas y el sistema, en el diseño de políticas educativas y en la formación en servicio de los docentes. Diversos estudios dan cuenta que las actitudes, creencias y valoraciones que los miembros de la comunidad educativa desarrollan hacia la democracia y la ciudadanía no han logrado construirse fuera de la formalidad de estos conceptos y lejos de su dimensión institucional. El objetivo es identificar aquellos puntos de desencuentro entre los documentos curriculares oficiales de formación inicial docente de institutos de educación superior pedagógica (IESP) en la especialidad de primaria y las actitudes de los docentes hacia la democracia y la ciudadanía. Los resultados evidenciaron que existen desencuentros entre la formación docente esperada y las actitudes de los profesores hacia la democracia.
\end{abstract}

Descriptores: Formación docente, Educación ciudadana, Currículo, Democracia, Justicia social.

With the return to democracy in 2001, the interest of the Peruvian education system in citizenship education and democracy was reflected in the incorporation of these subjects into the curricula of basic training and in the management of schools. Additionally, these topics were included in the design of the educational policy system and formed part of the in-service training of teachers. Several studies report that the attitudes, beliefs and values of the members of the educational community about democracy and citizenship have failed because these concepts have not been build outside of the formal definition or away from its institutional dimension. The main objective of this article is to identify those points of disagreement from the analysis of the official curriculum of initial teacher training in the specialty of primary education in Pedagogical Institutes (IESP). Moreover, it analyses the attitudes of teachers towards democracy and citizenship. The results show that there are disagreements between the expected teacher education and their attitudes towards democracy.

*Contacto: rcuenca@iep.org.pe

ISSN: 2254-3139

www.rinace.net/riejs/

revistas.uam.es/riejs
Recibido: $\quad 16$ de septiembre 2016

$1^{\text {a }}$ Evaluación: 24 de octubre 2016

Aceptado: $\quad 3$ de noviembre 2016 
Keywords: Teacher education, Citizenship education, Curriculum, Democracy, Social justice.

Com o regresso da democracia em 2001, o interesse do sistema educativo peruano pela formação cidadã e a democracia foi refletido na incorporação destes temas nas estruturas curriculares da formação básica, na gestão das escolas e do sistema, na elaboração de políticas educativas e na formação em serviço dos docentes. No entanto, diversos estudos mostram que as atitudes, crenças e valores que os membros da comunidade educativa desenvolvem frente à democracia e a cidadania foram construídos fora da formalidade destes conceitos e longe de sua dimensão institucional. $\mathrm{O}$ objetivo principal é identificar aqueles pontos de desencontro entre os documentos curriculares oficiais da formação inicial docente de institutos de educação superior pedagógica (IESP) na especialidade de primária e as atitudes dos docentes frente à democracia e a cidadania. De maneira geral, os resultados evidenciaram que existem desencontros entre a formação docente esperada e as atitudes dos professores frente à democracia.

Palavras-chave: Formação docente, Educação cidadã, Currículo, Democracia, Justiça social.

Este artículo se desarrolló en el marco del proyecto de investigación "Trayectorias educativas, juventudes y desigualdades sociales” auspiciado por la Fundación Ford.

\section{Introducción}

En el Perú, el regreso a la democracia en el año 2001 generó un especial entusiasmo entre la comunidad educativa. El signo de estos nuevos tiempos se expresó en el desarrollo de una agenda democratizadora en las más importantes dimensiones educativas. Tanto en las políticas como en el sistema, la gestión instaló nuevos mecanismos y espacios de participación, en donde la consulta y la búsqueda de consensos caracterizaron a los principales instrumentos de política educativa e instancias de gobierno del sistema ${ }^{1}$ (Cuenca, 2013; Oliart, 2011).

Por el lado pedagógico, el ejercicio democrático no solo se incorporó en la vida de las escuelas, sino que la formación ciudadana se constituyó en el centro de las discusiones curriculares y didácticas. El informe final de la Comisión de la Verdad y la Reconciliación (CVR) recomendó, por ejemplo, establecer un plan de estudios que estimule el conocimiento de las diferencias, la problematización de la realidad peruana y los principios democráticos para alejarse, de este modo, de la proclividad hacia la violencia que pone en riesgo el sistema democrático (Sandoval, 2004).

Desde el Ministerio de Educación se realizaron esfuerzos por incluir nociones vinculadas a la democracia y la ciudadanía. De hecho, el ejercicio de la ciudadanía, basada en el derecho y los deberes, así como en el respeto a la diversidad, fue uno de los cuatro objetivos planteados en el diseño curricular nacional para la educación básica aprobado en el año 2005 y, en la misma línea, es posible observar la incorporación explícita del tema en los documentos curriculares modificados de los años 2009 y 2016.

\footnotetext{
1 Al campo de las políticas corresponden el Acuerdo Nacional por la Educación (2002), la Ley General de Educación N ${ }^{\circ}$ 28044 (2003), el Pacto Social de Compromisos Recíprocos por la Educación (2004) y el Proyecto Educativo Nacional (2005). En relación al sistema hacemos referencia a los Consejos Participativos Regionales y Locales (Copare y Copale) así como a los Institucionales (Conei), entre otros.
} 
La formación en servicio para docentes ha introducido el tema de ciudadanía como un programa de especialización para el desarrollo del área curricular en el nivel de secundaria (2010) y como un programa de actualización en didáctica para primaria y secundaria (2013) e inicial (2014). ${ }^{2}$

No obstante, varios estudios (Ginocchio, Frisancho y La Rosa, 2015; Muñoz, 2009; Muñoz, Sempe y Vargas, 2015; Rodríguez y Domínguez, 2009; Stojnic, 2009 y 2015; Stojnic y Carrillo, 2016; Stojnic y Román, 2016; Ugarte, 2015; Yep y Barea, 2012) dan cuenta que las buenas intenciones, la nueva normatividad, la incorporación del tema en los documentos curriculares y su aplicación en las aulas no han logrado obtener mayores logros.

En el ámbito de la gestión, los espacios y mecanismos de participación no han podido consolidarse debido a que el enfoque de la participación que se propone no ha sido del todo claro ni consistente (Muñoz, Sempe y Vargas, 2015), a la pérdida de referentes claros de trabajo para los espacios institucionales de participación (Yep y Barea, 2012) y a la existencia de asuntos estructurales como el poco desarrollo del ejercicio ciudadano para responder a exigencias de la participación (Muñoz, 2009).

En las aulas, si bien el Informe Latinoamericano del Iccs del 2009 (Schulz, Ainley, Friedman y Lietz, 2011) presentó evidencia suficiente de que mayores niveles de conocimiento cívico y ciudadano entre estudiantes estaban correlacionados con un apoyo más fuerte hacia la democracia, en el caso peruano, Stojnic (2009, 2015) muestra que limitar o facilitar, en la cotidianeidad escolar, las posibilidades de que los estudiantes asuman responsabilidades y participen activamente en procesos relevantes para la institución educativa influiría de manera diferenciada en su valoración respecto a la democracia como sistema político, así como el rol que la ciudadanía debía tener en la misma. Y es que, como afirman Rodríguez y Domínguez (2009) para el caso de estudiantes de secundaria, la participación en las organizaciones escolares es fundamentalmente simbólica; es decir, sin mayor significado.

Ginocchio, Frisancho y La Rosa (2015) sostienen que el proceso de construcción de la democracia en el colegio tiene como actor principal al docente, cuyas concepciones y creencias influirán en su práctica pedagógica. Los resultados de su estudio revelaron que, si bien los docentes identificaron ventajas de vivir en un sistema democrático, no se mostraron convencidos de la efectividad de dicho sistema en su calidad de vida. Un ejemplo de ello es como los docentes justificaron el uso de "mano dura" para la aplicación de normas que no son respetadas por los ciudadanos. Estos mostraron que los profesores no reconocen la construcción de una sociedad democrática como un fin de la educación, sino que perciben que su objetivo principal es formar emprendedores preparados para el mundo laboral.

En ese sentido, Ugarte (2015) plantea que es importante fomentar no solo el desarrollo de capacidades técnicas en las instituciones educativas sino también competencias

${ }^{2}$ Como señalan Montero y Carrillo (2015), la oferta de formación en servicio por parte del Estado en las últimas décadas se ha venido ofreciendo a través de programas nacionales masivos, tales como el Plan Nacional de Capacitación Docente (Plancad, 1995-2001), el Programa Nacional de Formación en Servicio (PNFS, 2002-2006), el Programa Nacional de Formación y Capacitación Permanente (Pronafcap, 2007-2011), el Pronafcap Especializaciones (2011-2013), Programas de Especialización y Actualización Docente (2013-2014) y el Plan de Actualización Docente en Didáctica (Padd, 20142016). 
socioemocionales, comunicacionales y ciudadanas las cuales, si bien son valoradas por el sector laboral contemporáneo, también contribuyen a un ejercicio ciudadano pleno.

Es en este contexto que nos preguntamos: ¿cuál ha sido el papel de la formación inicial docente en estas dos décadas de cambios y transformaciones democráticas en nuestro país?, ¿incorpora en sus enfoques, perfiles y contenidos curriculares el desarrollo de las competencias democráticas y ciudadanas?, ¿qué se espera de los docentes que provienen de los institutos de educación superior pedagógica (IESP) en cuanto estos temas para contribuir a construir un país con mayor justicia social?

Es así que planteamos como hipótesis de trabajo que existe un desencuentro entre el ideal normativo construido alrededor de la educación ciudadana en la formación docente y las actitudes de los profesores frente a la democracia y la ciudadanía. De este modo, el artículo tiene como objetivo identificar aquellos puntos de desencuentro, a partir de un análisis de los documentos curriculares oficiales de formación inicial docente de IESP ${ }^{3}$ en la especialidad de primaria de los últimos veinte años, y la revisión de las opiniones de los docentes sobre la democracia y la ciudadanía. Para ello se seguirá el enfoque metodológico propuesto por Axel Honneth (2009) sobre cómo los desencuentros entre ideales y normas se constituyen en una patología social.

El desarrollo de competencias democráticas, de tolerancia política y de ejercicio de ciudadanía requiere un trabajo pedagógico intencional entre los docentes, pues estos no reconocen necesariamente la construcción de una sociedad democrática como un fin de la educación (Ginocchio, Frisancho y La Rosa, 2015). En tal sentido, si bien el paso por la educación formal contribuye a desarrollar dichas competencias, se requiere incorporarlas como parte de la noción de calidad (Stojnic y Carrillo, 2016) y como un contenido curricular (Stojnic y Román, 2016) para que efectivamente logren instalarse como aprendizajes.

\section{Marco de referencia}

Nuestro análisis utiliza como aproximación conceptual el reconocimiento de que el ejercicio pleno de una ciudadanía, complejizada a partir de las nociones clásicas de ciudadanía liberal, es una forma de participación que contribuye o se acerca a la justicia social. En este proceso de formación de ciudadanía, la educación, en general, y la escuela, en particular, juegan un papel fundamental. Tal como lo sostiene Apple (1996), la educación es, por naturaleza, un proceso ético y político encargado de formar "criaturas del Estado" (Bourdieu, 1997).

Para ello nos serviremos del constructo de justicia social desarrollado por Nancy Fraser (1995, 2006, 2008) y las propuestas de ciudadanía diferenciada impulsadas desde la filosofía feminista, particularmente en la desarrollada por Elizabeth Jelin (1997) e Iris Marion Young (1990). Desde nuestra perspectiva, las nociones de ciudadanía y de justicia social se encuentran directamente conectadas ya que, como veremos a continuación, tanto para Fraser como para Jelin y Young una condición clave para que

\footnotetext{
${ }^{3}$ En el Perú, la formación docente se imparte en tres tipos de centros de formación inicial: los institutos de educación superior pedagógica (IESP), las universidades y las escuelas de formación (artística o deportiva). El Estado solo puede intervenir en los planes de estudio de los institutos y escuelas, los cuales pueden ser públicos o privados y se distribuyen en las 25 regiones del país. Para el 2015, el total de IESP a nivel nacional era de 197 (81 privados y 116 públicos) con un total de 25793 estudiantes matriculados (Fuente: Censo Escolar 2015. Disponible en http://escale.minedu.gob.pe/).
} 
exista justicia social y ciudadanía es la participación de todos los grupos sociales en la vida pública y política del país.

Para Fraser (2006, 2008), la justicia social debe ser abordada como una conjunción entre las demandas de redistribución y reconocimiento. Es decir, constituye una categoría bidimensional, ya que no se puede entender sin integrar ambos tipos de reivindicación. Las demandas en materia de redistribución, por un lado, hacen referencia a la distribución equitativa de recursos y bienes entre los individuos y las sociedades, y, por el otro, las demandas de reconocimiento reclaman los aspectos relacionados con la identidad de las minorías "raciales", sexuales y étnicas.

En ese sentido, a través de la integración de ambas demandas, se afirman las disparidades que se encuentran en las sociedades tanto a nivel material como simbólico. La inequidad en la redistribución y la falta de reconocimiento, según lo señalado por Fraser (1995), produce que ciertos grupos se encuentren poco o nada representados en la arena política.

No obstante, en los años siguientes Fraser incorpora una dimensión política a sus reflexiones sobre justicia social. Sostiene que se requiere teoría que: "conceptualice la representación, junto con la distribución y el reconocimiento, como una de las tres dimensiones fundamentales de la justicia" (Fraser, 2008, p. 43). De este modo, la justicia social se presenta como el ideal contrafáctico de las desigualdades sociales (Cuenca, 2011).

Por este motivo, la justicia social necesita de la participación, ya que el principal elemento para lograr la distribución y el reconocimiento es lograr intervenir en la estructura de toma de decisiones (Murillo y Hernández-Castilla, 2011).

Este marco es propicio para las resignificaciones en la noción de ciudadanía. El interés por la ciudadanía asociada al acceso a una situación igualitaria a partir del ejercicio de un conjunto de derechos civiles, políticos y culturales (Marshall, 1950) se ha trasladado hacia una ciudadanía asentada en el valor de la diferencia y la importancia del reconocimiento, definiéndose no solo como un estatus legal, bajo los parámetros de derechos y deberes, sino también como un asunto de identidad (Young, 1990).

En esta línea, Jelin (1997) señala que la representación en los espacios públicos requiere de una noción de ciudadanía que se distancie del conjunto de prácticas concretas, tales como votar en los procesos electorales o recibir beneficios sociales de parte del Estado. Desde esta perspectiva, la ciudadanía es el eslabón que permite negociar, en una relación de poder, la definición de los problemas sociales comunes y cómo estos serán afrontados. Es decir, se ejerce a través de la participación activa en los espacios políticos y de gestión pública; aquello que se denomina, desde el enfoque de justicia social, "paridad de participación” y que permite integrar las demandas de distribución y reconocimiento (Fraser, 2006, 2008).

En tal sentido, la ciudadanía se encuentra directamente relacionada con la democracia, ya que la principal premisa de este sistema es que todos los ciudadanos son iguales ante la ley y, por ende, deben tener la misma capacidad de participar y ser representados en el sistema político. Como señala Remy (2011), en democracia, los ciudadanos tienen la facultad de tomar decisiones de manera equitativa, es decir, se funda bajo un sustento igualitario. Al respecto, Stojnic y Carrillo (2016) puntualizan en el consenso que existe al señalar la necesaria relación entre educación y el proceso de construcción democrática. 
Con relación a esto, indican que las instituciones educativas son concebidas como aquellos lugares de reproducción de valores y prácticas democráticas, es decir, son espacios idóneos para el desarrollo de actitudes ciudadanas.

Retomando todo lo dicho anteriormente, la discusión sobre cuáles son las posibilidades y los límites que presentan los diversos enfoques hegemónicos sobre la igualdad de oportunidades y la calidad utilitarista en el papel de los sistemas educativos en la construcción de justicia social parecen ausentes (Cuenca, 2012). Particularmente, esta discusión no forma parte de la investigación sobre temas de formación docente.

Si bien existen en el Perú diversos estudios sobre la formación inicial docente (Alcázar y Balcázar, 2001; Ames y Ucceli, 2008; Arregui, Hunt y Díaz, 1996; Burga, 2004; Escobar, Küper y Valiente-Catter, 1999; López de Castilla, 2004; Montero, Ames, Uccelli y Cabrera, 2005; Oliart, 1996; Ríos, 2007), las investigaciones respecto a la relación que existe entre esta etapa de formación y la ciudadanía son aún escasas. Recientemente, Iguíniz (2016) elaboró un informe sobre la formación inicial docente para la ciudadanía en el Perú. Dicho estudio fue realizado tanto en universidades como en IESP y, a través de este, hace un análisis documental de los diseños curriculares para la carrera del profesor. De la revisión de los planes de estudio de once instituciones seleccionadas concluye que el tratamiento de la educación para la ciudadanía es desigual en relación a los enfoques curriculares y los planes de estudio.

No obstante, se han encontrado aproximaciones a esta problemática en otros países. Por un lado, desde la perspectiva de las representaciones sociales, Cárcamo (2008) realizó un estudio entre los estudiantes y docentes universitarios de las carreras de pedagogía y educación en una universidad de Chile sobre la noción de ciudadanía que se maneja en este espacio y la importancia que se le atribuye en la formación inicial docente. Al respecto, señaló que existe una valoración positiva sobre la enseñanza de la ciudadanía en la formación inicial pero que, sin embargo, solo desarrolla en las asignaturas que corresponden al área pedagógica y no en aquellas vinculadas al desarrollo disciplinario. Asimismo, encontró que algunos académicos solo se refieren a la ciudadanía en términos discursivos más no se reflejan en actividades prácticas en el aula (Cárcamo, 2008).

Bajo este mismo marco de interpretación, Cárcamo (2010) realizó una investigación en una universidad pública de Madrid en la cual se pregunta sobre la influencia de la formación inicial docente sobre las representaciones sociales que posee el estudiantado sobre la ciudadanía y la formación ciudadana en la escuela. Por medio de la información recolectada, el autor encontró que en este espacio perdura una mirada de la ciudadanía que se restringe a aspectos procedimentales como el civismo o el derecho a participar en elecciones. Es decir, la formación inicial docente busca inculcar estrategias técnicopedagógicas para la trasmisión de contenidos referidos a la ciudadanía, llámese "educación cívica" o "educación en valores" pero, sin embargo, "se imparte con la finalidad de que aprenda a enseñar cómo formar la ciudadanía en la escuela en base de un conjunto de contenidos impuestos por Ley" (Cárcamo, 2010, p. 12).

En esta línea, Redón y Rubio (2006) realizan una reflexión teórica sobre la conexión entre las nociones de pedagogía, ciudadanía y formación docente. A través de esta, señalan que el desafío pedagógico se encuentra en la construcción de la ciudadanía, es decir, en hacer de la escuela un espacio civil, político y cultural que permita desarrollar personas que se confronten con la vivencia democrática. Asimismo, Jakubowicz, Ramos y Rodríguez (2011) plantean que la democratización y politización de los espacios de 
formación docente son fundamentales para la construcción de una ciudadanía como práctica emancipadora porque "son los docentes quienes, luego en sus aulas, tendrán la oportunidad de generar las condiciones de resistencia, de construir el espacio democrático, donde la ciudadanía se hace, se practica, y no sólo se tiene” (2011, p. 12). De esta forma, retoman lo señalado por Cantero (2006), quien indica que la ciudadanía debe lograrse a través de una actitud de descentramiento cultural, de una disposición de escucha sobre las necesidades de los estudiantes y sus familias, de una aproximación a las instituciones y movimientos sociales del entorno y, por último, al estímulo de la palabra como un instrumento de poder.

\section{Método}

El enfoque metodológico seleccionado es cualitativo y con una aproximación desde la teoría crítica. Combina, por un lado, la propuesta teórico-metodológica desarrollada por Axel Honneth $(2009,2014)$ para identificar los desencuentros entre ideales normativos y la praxis. El autor sostiene que estos desencuentros se constituyen en "patologías sociales"; es decir, desvíos en los que incurren las instituciones respecto a sus propios fines. En este sentido, el ideal normativo es aquel dispositivo que rige los lineamientos para el "correcto" funcionamiento de las instituciones y, por ende, establece los principios que rigen a los individuos que la conforman. De esta forma, las patologías sociales se encuentran en donde las instituciones sociales no cumplen con los fines para las que fueron diseñadas.

En este contexto, Honneth (2014) propone un marco metodológico para aproximarnos a dichas patologías. Plantea como procedimiento iniciar el análisis con la "reconstrucción normativa”, el cual constituye un instrumento para develar y analizar los ideales normativos existentes en las instituciones, para luego contrarrestar lo reconstruido con situaciones propias de la praxis.

Para nuestros fines, la reconstrucción normativa se realizará a partir del análisis descriptivo de los documentos curriculares de formación inicial docente de la especialidad de primaria de $1997 \mathrm{y}$, del vigente, de 2010. Para ello adaptamos un conjunto de matrices de análisis desarrolladas por Cox (2010) y Cox, Jaramillo y Reimers (2005).

Para identificar el tipo de aproximación que los currículos tienen hacia la educación ciudadana adaptamos la versión de Cox y otros (2005) que consigna información sobre el énfasis del marco curricular y los perfiles en tres ejes de análisis. El primero es un continuo entre la educación ciudadana tradicional, basada en el aprendizaje de aspectos de la institucionalidad política y la más actual visión de la educación ciudadana caracterizada por el énfasis en un doble foco: institucionalidad política y temas relacionales en las sociedades. El segundo eje tiene que ver con la ubicación de los contenidos en el plan de estudios hacia el final de la carrera o de manera longitudinal. Finalmente, el tercer eje centrado en si el enfoque curricular para el desarrollo de la educación ciudadana está centrado en conocimientos o en competencias que incluyan desarrollo de metodologías participativas. 
Un segundo tipo de análisis se realizará sobre la base de la adaptación de la matriz elaborada por Cox (2010) sobre el tipo de contenidos vinculados a la educación ciudadana ${ }^{4}$. De este modo, se identificaron seis categorías de análisis: (a) principios y valores cívicos (orientaciones que forman la base valorativa de la vida democrática), (b) ciudadanía y participación democrática (roles y relaciones de las personas con el orden político), (c) instituciones (instituciones referentes del sistema político democrático), (d) identidad pluralidad y diversidad (bases culturales y simbólicas en las relaciones sociales), (e) convivencia y paz (valores asociados al diálogo y a la resolución pacífica de conflictos) y (f) contexto macro (vínculos con la economía, la protección del ambiente y la globalización).

La identificación de los factores de la praxis se hizo a partir del análisis descriptivo de los resultados de una encuesta de opinión realizada por el Instituto de Estudios Peruanos y la Fundación Gustavo Mohme, para conocer percepciones y expectativas sobre ciudadanía y formación ciudadana de estudiantes, docentes y familias (Aragón, Cruz, de Belaunde, Eguren, González y Román, 2016). Dicha encuesta se realizó en octubre del 2015 y se aplicó a una muestra aleatoria de 22 escuelas públicas ubicadas en zonas urbanas marginales de las ciudades de Arequipa, Ayacucho, Iquitos, Lima, Piura, y de la Provincia Constitucional del Callao. El diseño muestral fue probabilístico estratificado basado en el registro de escuelas públicas, con aleatoriedad respecto al aula del grado seleccionado. Como resultado de la aplicación de en cuestas autoadministradas, se obtuvo una muestra conformada por 1356 estudiantes de sexto grado de primaria, 1119 estudiantes de cuarto grado de secundaria, 518 padres de familia y 341 docentes. El tamaño de la muestra y el diseño muestral permiten realizar estimaciones con un margen de error de 2,5 puntos porcentuales y con un nivel de confianza del $95 \%$.

Con este enfoque metodológico propuesto no se busca establecer relaciones causales o atribuciones directas al vínculo entre los currículos de formación y las opiniones de los docentes en servicio. El análisis sirve para aproximarnos al problema de investigación y construir nuevas hipótesis de trabajo. No obstante, el $78 \%$ de los docentes participantes provienen de un IESP y de ellos el 66\% fueron formados con los currículos analizados.

\section{Los desencuentros con la formación democrática y ciudadana}

Para este análisis se revisaron dos currículos de formación inicial docente: el Currículo Básico de Formación Docente de 1997 (en adelante CBFD) y el Diseño Curricular Básico Nacional de 2010 (en adelante DCBN). Ambos currículos cubren temporalmente un importante número de docentes formados que actualmente se desempeñan en las aulas.

El CBFD organiza una carrera que se estructura en tres etapas. La primera es de cuatro semestres y tiene que ver con la "teorización temprana" de la práctica. La segunda comprende los ciclos del quinto al octavo y prioriza el análisis y sistematización teórica a partir de la permanente interacción con la respectiva práctica. La tercera es la última etapa, que conduce al título profesional, y corresponde a los ciclos noveno y décimo en

${ }^{4}$ Cox se basa en una matriz elaborada para el International Civic and Citizenship Study (Iccs) del año 2009 elaborado por la Asociación Internacional para la Evaluación del Rendimiento Educativo (IEA). Más información en Schulz, Ainley, Friedman y Lietz (2011) 
los que se prioriza la estrecha relación entre la práctica intensiva y la investigación (Minedu, 1997).

El DCBN organiza la carrera docente en dos etapas. La primera se desarrolla en cuatro semestres y está orientada a una Formación General para lograr competencias. Esta etapa privilegia la formación disciplinar y ahonda la práctica de valores y el respeto a la diversidad. La segunda etapa comprende los semestres del quinto al décimo y se orienta a la Formación Especializada, es decir, desarrolla las competencias necesarias para el manejo de la especialidad ligada a la práctica educativa. Tiende a desarrollar el pensamiento creativo, crítico y complejo, al mismo tiempo que busca consolidar el juicio moral autónomo y el compromiso con la educación (Minedu, 2010).

\subsection{El análisis de los documentos curriculares de la formación docente}

Un primer análisis, basado en la metodología de Cox et al. (2005) (ver tabla 1), evidencia que el foco, tanto en el enfoque u orientación curricular como en el perfil de egreso del CBFD, es la educación ciudadana tradicional, basada en el aprendizaje de aspectos de la institucionalidad política. Sin embargo, el DCBN evidencia una visión más actual de la educación ciudadana caracterizada por el énfasis en un doble foco: institucionalidad política y temas relacionales en las sociedades.

Asimismo, en el segundo eje, relacionado a la ubicación de los contenidos en el plan de estudios, en el CBFD se menciona la transversalidad sin hacerlo evidente en los cursos del plan de estudios, mientras que en el DCBN la ciudadanía se desarrolla en los primeros años de formación básica, con énfasis en el desarrollo de los contenidos. Al contrario de lo que señalan Cox et al. (2005), no se identifican los cursos hacia el final de la carrera ni el tratamiento del tema de manera longitudinal a lo largo del plan de estudios.

Finalmente, el tercer eje, centrado en el enfoque curricular para el desarrollo de la educación ciudadana, se identifica que el CBFD está centrado principalmente en conocimientos mientras que el DCBN se centra en competencias, aunque estas no incluyen desarrollo de metodologías participativas adaptadas a la educación superior.

Para el caso de los documentos curriculares de la formación docente se observa que el paso de 1997 al 2010 es un tránsito hacia formas más complejas de entender la educación ciudadana. Siguiendo la clasificación de Akar (2012), hay una aproximación a un abordaje maximalista de la educación para la ciudadanía; es decir, un enfoque que reconoce la participación activa de los ciudadanos en la vida democrática y no solo la versión "mínima" de participación, consistente en el derecho a votar y el deber de cumplir las leyes. No obstante, tampoco es posible determinar que los documentos curriculares del 2010 se encuentren íntegramente orientados a dicho enfoque.

Por otro lado, un segundo tipo de análisis se basa en la metodología elaborada por Cox (2010) (ver tabla 2) sobre el tipo de contenidos vinculados a la educación ciudadana. Así se identifica que en el CBFD no se desarrollan los temas de ciudadanía y participación democrática ni sobre convivencia y paz. El énfasis es más bien sobre la educación cívica y la institucionalidad (instituciones; identidad, pluralidad y diversidad; contexto macro) en una sola subárea (Estudios teórico-prácticos de la sociedad) y sobre principios y valores éticos en otra (Proyectos de práctica social); mientras que el DCBN desarrolla los temas de ciudadanía planteados en las seis categorías en cuatro cursos de la educación básica (Ciencias sociales I y II, Diversidad y educación inclusiva, Educación intercultural) aunque se identifica claramente la poca articulación de estos temas en los cursos identificados en el plan de estudios. 
Tabla 1. Énfasis del marco curricular y los perfiles según foco, ubicación y aprendizajes

\begin{tabular}{|c|c|c|}
\hline \multicolumn{3}{|l|}{ Año 2007} \\
\hline \multirow{3}{*}{ Foco } & $\begin{array}{l}\text { El currículo está orientado hacia las competencias. } \\
\text { Promueve una formación más integral al incluir } \\
\text { contenidos conceptuales, procedimentales y } \\
\text { actitudinales. }\end{array}$ & $\begin{array}{l}\text { Facilitador: Brinda afecto, seguridad y confianza. Practica la tolerancia y la búsqueda de } \\
\text { consensos. Establece relaciones humanas dialogales a diferente nivel: interpersonal, intra e } \\
\text { interinstitucional, en el trabajo en equipo. Promueve relaciones humanizantes de género, } \\
\text { familiares y comunitarias. Vive los valores religiosos de su propia confesión y respeta los de } \\
\text { otras confesiones. Genera respuestas adecuadas para el bienestar colectivo y la defensa civil. } \\
\text { Identifica con su nación y promueve valores patrióticos, la soberanía y defensa nacional. }\end{array}$ \\
\hline & $\begin{array}{l}\text { Una orientación social intensa a lo largo de toda la } \\
\text { carrera, en la que se especifican momentos de contacto e } \\
\text { interrelación con la comunidad local, se privilegia una } \\
\text { intensa formación en valores y se introduce al análisis de } \\
\text { la sociedad global que empieza a envolvernos a todos. }\end{array}$ & $\begin{array}{l}\text { Investigador: Respeta el pensamiento divergente y valora la interculturalidad. Analiza e } \\
\text { interpreta, en equipo multidisciplinario, la realidad compleja, para plantear alternativas de } \\
\text { solución. Coordina con especialistas afines y promueve el intercambio de saberes. Adquiere } \\
\text { Instrumentos para comunicarse adecuadamente con una sociedad global compleja a través de } \\
\text { una Lengua Extranjera y de la Informática. }\end{array}$ \\
\hline & $\begin{array}{l}\text { El currículo de Formación Docente incluye una formación } \\
\text { cuidadosa de tipo intercultural, de género, ambiental, en } \\
\text { Derechos Humanos y similares. }\end{array}$ & $\begin{array}{l}\text { Promotor: Reconoce, practica y divulga la defensa de la salud, de los recursos naturales, de los } \\
\text { derechos humanos y de la paz. Practica y fomenta la responsabilidad solidaria, la participación y } \\
\text { la equidad. Se compromete con los problemas y aspiraciones de los demás. Favorece la } \\
\text { concertación, la organicidad y la institucionalidad democrática. }\end{array}$ \\
\hline Ubicación & $\begin{array}{l}\text { Los contenidos del área de Sociedad están distribuidos a lo } \\
\text { largo de la carrera. Se inician en el primer ciclo y } \\
\text { continúan hasta el octavo. Los dos últimos (noveno y } \\
\text { décimo) están reservados exclusivamente a la práctica y } \\
\text { la investigación. ( } 36 \text { horas } \mid 26 \text { créditos) }\end{array}$ & \\
\hline Aprendizaje & & $\begin{array}{l}\text { Macrocompetencia del área de sociedad: Conoce y utiliza instrumentos teórico-técnicos } \\
\text { actualizados, propios de las Ciencias Sociales que, a la vez que lo desarrollan integralmente como } \\
\text { persona, le permiten operar sobre ella, personal y/o colectivamente, para resolver problemas } \\
\text { desde una perspectiva plenamente humana y facilitar los respectivos aprendizajes en sus futuros } \\
\text { alumnos. } \\
\text { Competencia**: Compromiso permanente con el mejoramiento de la comunidad cercana, nacional } \\
\text { y planetaria, desarrolla la propia personalidad en términos de autoestima, identidad, valores, } \\
\text { actitudes positivas, afectividad en general; y ejercita una vida democrática habiendo desarrollado } \\
\text { las destrezas operativas en cada caso, sobre la base de un juicio moral autónomo y en el marco de } \\
\text { una nueva ética. }\end{array}$ \\
\hline
\end{tabular}


Orienta el desarrollo de la formación ciudadana en el marco del respeto a los derechos humanos y la tolerancia antes las diferencias, favoreciendo la convivencia democrática y la construcción de la paz con justicia social
Humanista: Propicia una educación que fomente el desarrollo y crecimiento integral del ser humano para que se involucre como agente activo en la construcción de una sociedad donde confluyan la paz, la libertad y la solidaridad universal; un profesional que se forme bajo un marco nacional e internacional, a través del estudio de problemas mundiales contemporáneos, retos cruciales para la humanidad; respeto a los derechos humanos, protección del ambiente y promoción de la cooperación entre naciones.

Intercultural: Concibe la diferencia como una cualidad que implica comprensión y respeto recíproco entre distintas culturas; así como una relación de intercambio de conocimientos y valores en condiciones de igualdad, aportando al desarrollo del conocimiento, de la filosofía y cosmovisión del mundo y a las relaciones que en éste se establecen entre diferentes actores, en diferentes circunstancias. Permite asumir una conciencia crítica de la propia cultura y afrontar en mejores términos la globalización y mundialización.

Ambiental: Plantea el desarrollo sostenible desde la ética de la responsabilidad y solidaridad que debe existir entre los seres humanos y entre estos y el resto de la naturaleza, es decir, desde una óptica intra e intergeneracional, desde una línea biocenocéntrica. Según este enfoque, la "comunidad ética" se entiende como una comunidad que se interesa no sólo por el hombre (antropocentrismo) sino por los seres vivos en su conjunto, sin descuidar la naturaleza inanimada.

De equidad e inclusión: Se basa en la igualdad esencial entre los seres humanos, la cual se concretiza en una igualdad real de derechos y poderes socialmente ejercidos. Reconoce la necesidad de igualdad de oportunidades en el acceso y permanencia; exige trato de calidad sin distinción de etnia, religión, género u otra causa de discriminación.

Cultura de paz y respeto a los derechos ciudadanos: Supone un cambio de mentalidad individual y colectiva desde las aulas, en las que el profesor promueve la construcción de valores que permitan una evolución del pensamiento social; con un respeto irrestricto a la democracia, a los derechos humanos, a la libertad de conciencia, de pensamiento, de opinión, al ejercicio pleno de la ciudadanía y al reconocimiento de la voluntad popular; que contribuye a la tolerancia mutua en las relaciones entre las personas, entre las mayorías y minorías y en el fortalecimiento del Estado de Derecho. 


\section{ENFOQUE/ORIENTACIÓN}

PERFIL DE EGRESO

Los cursos de Ciencias sociales (I y II), Diversidad y

Ubicación educación inclusiva y Educación intercultural está

horas/13 créditos)

Aprendizaje

Competencia global (dimensión personal): Gestiona su autoformación permanente y practica la ética en su quehacer, estableciendo relaciones humanas de respeto y valoración, para enriquecer su identidad, desarrollarse de manera integral y proyectarse socialmente a la promoción de la dignidad humana.

Unidad de competencia: Demuestra conducta ética con responsabilidad y compromiso en los escenarios en los que se desenvuelve para fortalecer su identidad.

Competencia global (dimensión socio-comunitaria): Actúa como agente social, con respeto y valoración por la pluralidad lingüística y de cosmovisiones, para aprehender significativamente la cultura, gestionar proyectos institucionales y comunitarios, a fin de elevar la calidad de vida desde el enfoque de desarrollo humano.

Unidad de competencia: Interactúa con otros actores educativos de manera armónica, constructiva, crítica y reflexiva generando acciones que impulsen el desarrollo institucional.

Unidad de competencia: Interactúa socialmente demostrando conocimiento y comprensión por la diversidad lingüística y cultural, valorando la diferencia y la especificidad como un derecho humano.

Unidad de competencia: Desarrolla proyectos comunitarios en alianza con diferentes instituciones gubernamentales y de la sociedad civil, a fin de incentivar la responsabilidad social, potenciar las posibilidades y oportunidades de equidad e inclusión social y de esta manera contribuir a mejorar la calidad de vida de la población con la que se vincula.

Nota: Todas las secciones de la matriz son trascripciones de los documentos curriculares oficiales. * Los perfiles de egreso en ambos currículos están organizados de manera distinta. En el caso del CBFD (1997) el perfil está organizado en cuatro saberes (saber ser, saber convivir, saber pensar y saber hacer) y tres roles (facilitador del aprendizaje, investigador y promotor de la comunidad). El DCBN (2010) se organiza en tres dimensiones (personal, profesional-pedagógica y socio-comunitaria) y cinco enfoques (humanista, intercultural, ambiental, de equidad e inclusión y cultura de paz y respeto a los derechos ciudadanos). ** Es una de las cuatro competencias que se vincula con los temas de democracia y ciudadanía.

Fuente: Elaboración propia. 
Tabla 2. Análisis del tipo de contenidos vinculados a la educación ciudadana

\begin{tabular}{|c|c|c|c|c|c|c|}
\hline & \multirow[b]{2}{*}{ CATEGORÍAS } & \multicolumn{5}{|c|}{ PLAN DE ESTUDIOS* } \\
\hline & & $\begin{array}{l}\text { Estudios } \\
\text { teórico- } \\
\text { prácticos de } \\
\text { la sociedad }\end{array}$ & $\begin{array}{l}\text { Proyectos de } \\
\text { práctica } \\
\text { social }\end{array}$ & $\begin{array}{l}\text { CCSS } \\
\text { I y II }\end{array}$ & $\begin{array}{c}\text { Diversidad y } \\
\text { Ed. } \\
\text { inclusiva }\end{array}$ & $\begin{array}{l}\text { Ed. } \\
\text { inter- } \\
\text { cultural }\end{array}$ \\
\hline \multirow{6}{*}{ 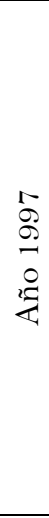 } & $\begin{array}{l}\text { Principios y } \\
\text { valores cívicos }\end{array}$ & & $\mathrm{X}$ & & & \\
\hline & $\begin{array}{c}\text { Ciudadanos y } \\
\text { participación } \\
\text { democrática }\end{array}$ & & & & & \\
\hline & Instituciones & $\mathrm{X}$ & & & & \\
\hline & $\begin{array}{l}\text { Identidad, } \\
\text { pluralidad y } \\
\text { diversidad }\end{array}$ & $\mathrm{X}$ & & & & \\
\hline & $\begin{array}{l}\text { Convivencia y } \\
\text { paz }\end{array}$ & & & & & \\
\hline & Contexto macro & $\mathrm{X}$ & & & & \\
\hline \multirow{6}{*}{ 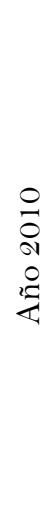 } & $\begin{array}{l}\text { Principios y } \\
\text { valores cívicos }\end{array}$ & & & $\mathrm{X}$ & & \\
\hline & $\begin{array}{l}\text { Ciudadanos y } \\
\text { participación } \\
\text { democrática }\end{array}$ & & & $\mathrm{X}$ & & \\
\hline & Instituciones & & & $\mathrm{X}$ & & \\
\hline & $\begin{array}{l}\text { Identidad, } \\
\text { pluralidad y } \\
\text { diversidad } \\
\end{array}$ & & & & $\mathrm{X}$ & $\mathrm{X}$ \\
\hline & $\begin{array}{l}\text { Convivencia y } \\
\text { paz }\end{array}$ & & & & $\mathrm{X}$ & $\mathrm{X}$ \\
\hline & Contexto macro & & & $\mathrm{X}$ & & \\
\hline
\end{tabular}

Nota: * Debido al diseño y organización del plan de estudios, para el CBFD de 1997 se presentan las subáreas y para el DCBN del 2010 se presentan cursos.

Fuente: Elaboración propia.

Asimismo, una mirada general al DCBN evidencia que este tiene un enfoque tradicional en el tema y el reconocimiento al otro es desde una mirada humanizante, en el que el docente está fuera del contexto y tiene que aprender a mirar, incorporar, valorar y respetar, "humanizar al otro" pero no se evidencia el involucrarse con el otro, reconocerlo como un igual en su condición de ciudadanos. Un ejemplo es el docente promotor, que más que un ciudadano dentro de un sistema democrático, el énfasis es un impulsor de proyectos para el desarrollo de la comunidad en la que trabaja, inclusive se propone hacerlo desde una mirada empresarial.

Mientras tanto, en el DCBN se puede identificar que si bien el plan de estudios mantiene un enfoque tradicional, en los primeros dos años de formación, dedicados a los estudios generales, se desarrollan cursos con contenidos sobre ciudadanía, pero estos están desarticulados entre sí, lo que puede hacer que la ciudadanía sea un tema fragmentado en cuanto contenidos, y en cuanto teoría con la realidad educativa y la práctica pedagógica porque se prioriza el aprendizaje de conceptos.

En resumen, las características muestran que si bien hay un avance entre el currículo de 1997 y el del 2010, en este último se presenta cierto nivel de inconsistencia entre las 
orientaciones curriculares y los planes de estudio. Aun cuando estos son presentados desde un enfoque de competencias, los planes muestran centralidad en los contenidos de la educación ciudadana. De este modo, el tránsito de la educación cívica a la educación ciudadana quedaría incompleto al no centrase en el desarrollo de habilidades y actitudes fundamentales para el desarrollo de competencias en ciudadanía (Osler y Starkey, 2006).

\subsection{Actitudes, creencias y percepciones sobre ciudadanía}

El reciente estudio del IEP y Fundación Mohme (Aragón et al., 2016) sobre la ciudadanía en la escuela ${ }^{5}$ encontró que, si bien existe una disposición positiva por parte de los docentes hacia la democracia como sistema político, esta se encuentra mayormente asociada al respeto de los derechos y deberes ciudadanos, así como a la igualdad de oportunidades. En tal sentido, el estudio evidenció que la mayoría de los docentes asocia el ejercicio de la ciudadanía al respeto de normas y derechos, más no lo relacionan con la participación política.

Como se puede apreciar en la tabla siguiente, el 67\% de los docentes encuestados indicó que una de las principales características de la democracia era el respeto a los derechos de todos los ciudadanos y un $54 \%$ señaló que era la igualdad de oportunidades. En contraparte, con relación a la participación activa de los ciudadanos frente a las decisiones de gobierno, los profesores mostraron una menor inclinación a la idea de que los ciudadanos puedan discutir e intervenir activamente sobre dichas decisiones. Al respecto, solo el $10 \%$ de los encuestados señaló como una de las principales características que los ciudadanos puedan influir en las decisiones del gobierno y el $15,8 \%$ indicó que las personas tengan la libertad de discutir sobre dichas decisiones.

Tabla 3. Características de la democracia: Existen distintas visiones sobre lo que es la democracia, ¿¿cuáles son las dos características más importantes de una democracia?

\begin{tabular}{|c|c|c|c|}
\hline RESPUESTAS & $\begin{array}{c}\text { TotAL } \\
(\%)\end{array}$ & HOMBRE & MUJER \\
\hline $\begin{array}{l}\text { Que los ciudadanos(as) puedan participar en las elecciones para } \\
\text { elegir a sus gobernantes }\end{array}$ & 22,4 & 19,4 & 23,5 \\
\hline $\begin{array}{l}\text { Que todas las personas tengan las mismas oportunidades sociales } \\
\text { y económicas }\end{array}$ & 54,0 & 54,8 & 53,7 \\
\hline $\begin{array}{l}\text { Que las decisiones del gobierno se tomen de acuerdo con lo que } \\
\text { quiere la mayoría }\end{array}$ & 27,4 & 22,6 & 29,1 \\
\hline $\begin{array}{l}\text { Que los ciudadanos(as) puedan influir activamente en las } \\
\text { decisiones del gobierno }\end{array}$ & 10,0 & 15,1 & 8,2 \\
\hline $\begin{array}{l}\text { Que los ciudadanos(as) tengan la libertad de discutir sobre las } \\
\text { decisiones del gobierno }\end{array}$ & 15,8 & 16,1 & 15,7 \\
\hline Que se respeten los derechos de todos los ciudadanos(as) & 66,8 & 69,9 & 65,7 \\
\hline $\mathrm{NS} / \mathrm{NR}$ & 3,3 & 2,2 & 3,7 \\
\hline
\end{tabular}

Fuente: IEP y Fundación Mohme (2016).

En esta línea, las formas como conciben el sistema democrático tienen un correlato con las características de un buen ciudadano señaladas por los docentes. Al respecto, el $58,2 \%$ indicó que un buen ciudadano es aquel que respeta las opiniones distintas a las de

\footnotetext{
5 Mayor información del "Proyecto Democracia: construyendo ciudadanía desde la escuela" disponible en: http://iep.org.pe/investigaciones-y-proyectos/proyecto-democracia-construyendo-ciudadania-desde-la-escuela/.
} 
uno, el 43,5\% señaló que es el que obedece siempre las leyes y normas y el 41,6\% indicó que un buen ciudadano es aquel que paga sus impuestos.

Tabla 4. Características del buen ciudadano: Existen diferentes opiniones sobre lo que es ser un buen ciudadano(a). Elija las TRES acciones que usted cree que son las más importantes para considerar a alguien un buen ciudadano(a)

\begin{tabular}{lrrr}
\hline \multicolumn{1}{c}{ RESPUESTAS } & $\begin{array}{r}\text { TOTAL } \\
\text { \% }\end{array}$ & HOMBRE & MUJER \\
\hline Respetar a la gente con opiniones distintas a la de uno & 58,2 & 58,1 & 58,2 \\
Vigilar que las autoridades públicas actúen correctamente & 23,8 & 28,0 & 22,4 \\
Obedecer siempre las leyes y normas & 43,5 & 43,0 & 43,7 \\
Ser trabajador & 24,9 & 22,6 & 25,7 \\
Realizar trabajo comunitario o voluntariado & 16,3 & 10,8 & 18,3 \\
Participar en una protesta contra una ley que se considere & 15,2 & 16,1 & 14,9 \\
$\quad$ injusta & 16,6 & 28,0 & 12,7 \\
Demostrar respeto por las autoridades & 5,5 & 2,2 & 6,7 \\
Seguir los temas políticos en el diario, la radio, la televisión o & & 26,9 & 25,0 \\
$\quad$ Internet & 25,5 & 2,2 & 1,1 \\
Conocer la historia del Perú & 1,4 & 2,4 \\
Unirse a un partido político & 41,6 & 37,6 & 42,9 \\
Pagar siempre sus impuestos & 24,9 & 21,5 & 26,1 \\
Votar siempre en las elecciones & 1,7 & 2,2 & 1,5 \\
NS/NR & & & \\
\hline Funte: IEP y Fun
\end{tabular}

Fuente: IEP y Fundación Mohme (2016).

Las respuestas relacionadas a la participación en protestas para modificar leyes o la afiliación a un partido político quedaron rezagadas con un $15,2 \%$ y un $1,4 \%$ respectivamente. Esto, a su vez, tiene relación con la respuesta respecto a su interés hacia la política, ya que la mayor parte de los docentes contestó tener algo o poco interés $(68 \%)$.

Cabe mencionar que a través de este estudio se evidenció que los docentes se encontraban a favor de medidas como el cierre del Congreso en caso de corrupción de congresistas $(78 \%)$ y al uso de militares para controlar la delincuencia (66\%). Estas respuestas muestran una actitud ambivalente hacia el sistema democrático, ya que se aceptan, en la mayoría de los casos, medidas de corte autoritario para casos específicos.

Por otro lado, en relación con el tema de la diversidad cultural, si bien la mayoría de los docentes se mostró de acuerdo con que cada quien debería tener derecho a educarse en su propia lengua, que la mezcla de personas y razas distintas era buena para el Perú y que cada pueblo debería conservar sus propias tradiciones y costumbres, aún el 19\%, $18 \%$ y $15 \%$ respectivamente se encuentra en desacuerdo con este tipo de aseveraciones.

Como se puede observar en la tabla 4, el estudio también muestra que alrededor del 40\% de los docentes piensa que los pobres son pobres porque no se esfuerzan lo suficiente y que el mismo porcentaje señaló que los pobres son pobres porque solo quieren recibir ayuda del Estado.

Recopilando, aquello que muestra el estudio es que aún los docentes cuentan con una visión más bien normativa de lo que es la ciudadanía, mas no han logrado incorporar las nociones de participación y representación en el espacio político como elementos fundamentales para el ejercicio ciudadano. Asimismo, como se pudo apreciar en los 
últimos ítems reseñados, aún existe un preocupante porcentaje de docentes que no percibe la diversidad como un valor importante y que, por el contrario, señalan como un elemento desfavorable la mezcla de "razas" y la conservación de culturas; de la misma manera que percibe la pobreza como una condición personal más que estructural (tabla $5)$.

Tabla 5. Percepciones sobre la pobreza

\begin{tabular}{lcccrrr}
\hline & \multicolumn{2}{c}{$\begin{array}{c}\text { Los pobres son pobres porque solo } \\
\text { quieren vivir de la ayuda del Estado }\end{array}$} & \multicolumn{4}{c}{$\begin{array}{c}\text { Los pobres son pobres porque no se } \\
\text { esfuerzan lo suficiente }\end{array}$} \\
\cline { 2 - 7 } & ToTAL \% & HOMBRE & MUJER & TOTAL \% & HOMBRE & MUJER \\
\hline Muy de acuerdo & 13,0 & 12,9 & 13,1 & 9,1 & 10,8 & 8,6 \\
De acuerdo & 29,6 & 22,6 & 32,1 & 33,2 & 29,0 & 34,7 \\
En desacuerdo & 46,3 & 53,8 & 43,7 & 46,5 & 47,3 & 46,3 \\
Muy en desacuerdo & 10,5 & 10,8 & 10,4 & 10,8 & 12,9 & 10,1 \\
NS/NR & 0,6 & 0,0 & 0,7 & 0,3 & 0,0 & 0,4 \\
\hline
\end{tabular}

Fuente: IEP y Fundación Mohme (2016).

\section{Reflexiones finales}

El objetivo de este artículo fue identificar aquellos puntos de desencuentro, a partir de un análisis de los documentos curriculares oficiales de formación inicial docente de IESP en la especialidad de primaria de los últimos veinte años y la revisión de las opiniones de los docentes en servicio sobre la democracia y la ciudadanía. Para ello se realizó un análisis descriptivo de los documentos curriculares y de las opiniones de los docentes de un estudio sobre actitudes hacia la ciudadanía y la democracia. Esta fue la base de trabajo para identificar dichos desencuentros, a partir del enfoque teórico-metodológico de patologías sociales.

Aun cuando este análisis es una aproximación inicial a cómo la formación docente aborda el tema de democracia y ciudadanía, los hallazgos de este estudio muestran que existe un desalineamiento entre el ideal normativo y las actitudes de los docentes. En términos de Honneth (2009, 2014) estamos ante una patología social.

Esta patología evidencia desviaciones de ese ideal, en este caso formativo, manifestado en una realización social con los resultados distintos a los esperados. Se produce una "falta de racionalidad" (Honneth, 2009, p. 31) pues las instituciones no se conducen en el camino que les permita alcanzar dichos ideales; es decir, no cumplen necesariamente con los fines para los cuales fueron diseñadas. En otras palabras, existen indicios de que el objetivo de formación en educación ciudadana y competencias democráticas no sea cumplido en la formación docente debido a factores propios de dichos procesos formativos.

Algunas posibles razones que explicarían esta patología pueden ubicarse en el plano estructural asociados a la poca tradición participativa en el Perú. Por ejemplo, los resultados del Barómetro de las Américas muestran que la participación ciudadana tanto en reuniones convocadas por la municipalidad como en la elaboración del presupuesto municipal "se ha mantenido estable y en niveles bastante modestos, la participación en reuniones convocadas por la municipalidad no supera el $15 \%$ y la participación en la elaboración del presupuesto municipal no supera el 4\%” (Carrión, Zárate y Zechmeister, 2015, p. 243). 
Sin embargo, algunas otras razones son posibles de hallarse en los propios procesos de formación docente, como el diseño y manejo curricular. Aun cuando el currículo vigente de formación docente (DCBN) está más cercano a la triple expansión-desarrollada por Cox, Bascopé, Castillo, Miranda y Bonhomme (2014) que consiste en la ampliación del foco de atención de la educación cívica a la ciudadana, de la característica longitudinal de los contenidos y la incorporación de actitudes y prácticas, además de conocimientos específicos-, falta fortalecer la técnica curricular para que permita de manera efectiva desarrollar competencias democráticas y ciudadanas que respondan a las exigencias actuales, más complejas y más cercanas a la interacción social que a los factores institucionales del Estado, el gobierno y la política.

Específicamente, los perfiles de egresados tienen un énfasis en la formación personal (dimensión personal) relacionada con los aspectos de la formación y la ética. Sin embargo, la intervención en la práctica pedagógica y el aula (dimensión profesionalpedagógica) instrumentaliza el quehacer del docente sin que exista alguna mención a procesos que democraticen la función en el aula.

Un aspecto particular es la presencia del tema religioso que se ocupa de desarrollar habilidades vinculadas a la tolerancia y la comprensión asociadas al ejercicio de convivencia. De alguna manera, se trata de "humanizar" a la "ciudadanía".

A pesar de que el DCBN (2010) incorpora la noción de ciudadanía, esta se encuentra más ligada a la tolerancia, entendida como respeto y reconocimiento de otro en su condición de ciudadano, más no a una mirada que reconoce la diversidad, pero también la incluye y la fomenta en los espacios de participación, tal como lo señala Fraser (2009) respecto a la conexión entre ciudadanía y justicia social.

En tal sentido, las recomendaciones de la Comisión de la Verdad y la Reconciliación proponen revisar las propuestas metodológicas y pedagógicas de la formación docente y reorientarlas para que brinden herramientas que permitan producir conocimiento y ejercitar la capacidad de reflexión y análisis crítico para el fortalecimiento de la democracia (CVR, 2003).

Por otro lado, los docentes cuentan con una noción de ciudadanía más ligada a los aspectos institucionales/normativos que a los relacionados a la representación política. En tal sentido y en relación con lo señalado por Jelin (1997), aún mantienen y reproducen una ciudadanía ligada a prácticas concretas, como el pago de impuestos o la votación, y no a aquellos aspectos que buscan negociar y disputar la representación en el marco de un sistema democrático.

Tomando en cuenta que la escuela es señalada como la institución idónea para el desarrollo de actitudes ciudadanas (Stojnic y Carrillo, 2016), cabría preguntarse hasta qué punto los formadores de docentes y los profesores en las aulas imprimen en sus estudiantes una mirada sobre la ciudadanía y la democracia más reglamentarista que participativa.

Finalmente, queda abierta la discusión sobre la necesidad de democratizar el íntegro del sistema educativo peruano para efectivamente formar ciudadanos que construyan de manera crítica un régimen y una sociedad democráticos. No ceder el espacio a la tentación de la educación rentista y las habilidades propias de la meritocracia es una tarea fundamental de las políticas y las reformas educativas, pues tal como lo sostiene 
Martha Nussbaum (2010), sin la intención de formar personas con pensamiento crítico y reflexivo, la democracia puede estar en peligro.

\section{Referencias}

Akar, B. (2012). The space between civic education and active citizenship in Lebanon. En M. Shuayb (Ed.), Rethinking education for social cohesion. International case studies (pp. 154-170). Londres: Palgrave Macmillan.

Alcázar, L. y Balcázar, R. A. (Coords.). (2001). Oferta y demanda de formación docente en el Perú. Lima: Ministerio de Educación.

Ames, P. y Uccelli, F. (2008). Formando futuros maestros; observando las aulas de institutos superiores pedagógicos públicos. En M. Benavides (Ed.), Análisis de programas, procesos y resultados educativos en el Perú. Contribuciones empiricas para el debate (pp. 131-175). Lima: Grade.

Apple, M. (1996). El conocimiento oficial. La educación democrática en una era conservadora. Buenos Aires: Paidós.

Aragón, J., Cruz, M., de Belaunde, C., Eguren, M., González, N. y Román, A. (2016). La ciudadanía desde la escuela. Encartes del Proyecto Democracia. Lima: Instituto de Estudios Peruanos y Fundación Gustavo Mohme Llona. Recuperado de http://iep.org.pe/investigaciones-y-proyectos/proyecto-democracia-construyendociudadania-desde-la-escuela/

Arregui, P., Hunt, B. y Díaz, H. (1996). Problemas, perspectivas y requerimientos de la formación magisterial en el Perú. Lima: Ministerio de Educación.

Bourdieu, P. (1997). Razones prácticas. Sobre la teoría de la acción. Barcelona: Anagrama.

Burga, E. (2004). Los procesos de aprendizaje en la formación docente: una mirada desde el nuevo enfoque pedagógico y la interculturalidad. Lima: Proeduca-GTZ.

Cantero, G. (2006). Educación popular en la escuela pública: Una esperanza que ha dejado de ser pura espera. Desde ciertos saberes, prácticas y condiciones. En P. Martinis y P. Redondo (Comps.), Igualdad y educación: escrituras entre dos orillas (pp. 205-223). Buenos Aires: Del Estante.

Cárcamo, H. (2008). Ciudadanía y formación inicial docente: Explorando las representaciones sociales de académicos y estudiantes. Revista de Pedagogía, 29(85), 245-268.

Cárcamo, H. (2010). Explorando las dimensiones constitutivas de la ciudadanía docente. Horizontes Educacionales, 15(2), 33-47.

Carrión, J., Zárate, P. y Zechmeister, E. (2015). Cultura política de la democracia en Perú y las Américas, 2014. Gobernabilidad democrática a través de 10 años del Barómetro de las Américas. Lima: LAPOP, IEP, USAID, Barómetro de las Américas y Vanderbilt University.

Cox, C., Bascopé, M., Castillo, J. C., Miranda, D. y Bonhomme, M. (2014). Educación ciudadana en América Latina: prioridades de los currículos escolares. Ginebra: Unesco-IBE.

Cox, C. (2010). Informe de referente regional 2010. Oportunidades de aprendizaje escolar de la ciudadanía en América Latina: Currículos comparados. Sistema Regional de Evaluación y Desarrollo de Competencias Ciudadanas (SREDECC). Bogotá: CERLALC.

Cox, C., Jaramillo, R. y Reimers, F. (2005). Educar para la ciudadanía y la democracia en las Américas. Una agenda para la acción. Washington D.C.: BID. 
Cuenca, R. (2011). Argumentos filosóficos sobre la desigualdad. Balance crítico de la noción. En J. Cotler y R. Cuenca (Eds.), Las desigualdades en el Perú. Balances críticos (pp. 31-58). Lima: Instituto de Estudios Peruanos.

Cuenca, R. (2012). Sobre justicia social y su relación con la educación en tiempos de desigualdad. Revista Internacional de Educación para la Justicia Social, 1(1), 79-63.

Cuenca, R. (2013). Cambio, continuidad y búsqueda de consenso, 1980-2011. Lima: Derrama Magisterial.

Comisión de la Verdad y la Reconciliación. (2003). Informe final. Tomo IX. Recomendaciones institucionales. Lima: Autor.

Escobar, N., Küper, W. y Valiente-Catter, T. (Eds.). (1999). Fórum internacional de formación docente. Miradas desde su práctica formativa. Lima: Ministerio de Educación y GTZ.

Fraser, N. (1995). From redistribution to recognition? Dilemmas of justice in a "post socialist" age. Recuperado de http://docplayer.net/385837-From-redistribution-to-recognitiondilemmas-of-justice-in-a-post-socialist-age.html

Fraser, N. (2006). La justicia social en la era de la política de la identidad: Redistribución, reconocimiento y participación. En N. Fraser y A. Honneth (Eds.), ¿̨Redistribución o reconocimiento? Un debate político filosófico (pp. 17-88). Madrid: Morata.

Fraser, N. (2008). Escalas de justicia. Barcelona: Herder.

Ginocchio, L., Frisancho, S. y La Rosa, M. I. (2015). Concepciones y creencias docentes sobre la democracia en el colegio. Revista Peruana de Investigación Educativa, 7(1), 5-29.

Honeth, A. (2009). Patologías de la razón. Historia y actualidad de la teoría crítica. Buenos Aires: Katz.

Honeth, A. (2014). El derecho de la libertad. Esbozo de una eticidad democrática. Buenos Aires: Katz.

Iguíñiz, M. (2016). La formación inicial de docentes en educación para la ciudadanía-Perú. Documento inédito. Santiago de Chile: Orealc-Unesco.

Jakubowicz, J., Ramos Gonzáles, J. y Rodríguez, F. (agosto, 2011). La crisis del concepto moderno de ciudadanía y la formación docente para la construcción de una ciudadanía emancipadora. Trabajo presentado en el VIII Encuentro de Cátedras de Pedagogía de Universidades Nacionales Argentinas "Teoría, formación e intervención en Pedagogía". La Plata: Universidad Nacional de La Plata.

Jelin, E. (1997). Igualdad y diferencia: dilemas de la ciudadanía de las mujeres en América Latina. Agora, Cuaderno de Estudios Políticos, 7, 189-214.

López de Castilla, M. (2004). La formación docente en Bolivia, Paraguay y Perú. Un análisis comparativo. En I. Flores (Ed.), ¿Cómo estamos formando a los maestros en América Latina? (pp. 103-116). Lima: Proeduca-GTZ y ORELAC-Unesco.

Marshall, T. (1950). Citizenship and social class, and other essays. Cambridge: Cambridge University Press.

Ministerio de Educación. (1997). Currículo básico de formación docente. Especialidad de Educación Primaria. Lima: Ministerio de Educación.

Ministerio de Educación. (2010). Diseño curricular básico nacional para la carrera profesional de profesor de educación primaria. Lima: Ministerio de Educación.

Montero, C. y Carrillo, S. (2015). Estudio sobre la oferta y calidad de los programas de formación docente en servicio del periodo 2011-2015. Informe final. Lima: Unesco Perú. 
Montero, C., Ames, P., Uccelli, F. y Cabrera, Z. (2005). Oferta, demanda y calidad en la formación de docentes. Los casos de Cajamarca, Cusco, Piura, San Martín y Tacna. Lima: Proeduca-GTZ.

Muñoz, F. (2009). Los caminos cruzados de la participación ciudadana en educación: Entre el mejoramiento de la calidad de la educación y la construcción de ciudadanía. Un estudio colectivo de casos en Piura. Revista Peruana de Investigación Educativa, 1(1), 31-60.

Muñoz, F., Sempé, L. y Vargas, J. (2015). Revisión de políticas públicas en educación, Perú (20002015). Lima: Unesco-Perú.

Murillo, J. y Hernández-Castilla, R. (2011). Hacia un concepto de justicia social. Revista Iberoamericana sobre Calidad, Eficacia y Cambio en Educación, 9(4), 7-23.

Nussbaum, M. (2010). Sin fines de lucro. Por qué la democracia necesita de las humanidades. Buenos Aires: Katz.

Oliart, P. (1996). ¿Amigos de los niños? Cultura académica en la formación del docente de primaria. Lima: Grade.

Oliart, P. (2011). Políticas educativas y la cultura del sistema escolar en el Perú. Lima: Instituto de Estudios Peruanos y Tarea.

Osler, A. y Starkey, H. (2006). Education for democratic citizenship: a review of research, policy and practice 1995-2005. Research Papers in Education, 24, 433-466.

Redón, S. y Rubio, G. (2006). Sujeto y pedagogía: ciudadanía y formación docente. Revista Iberoamericana de Educación, 40(2), 1-10.

Remy, M. I. (2011). Democracia y desigualdad: poderes fácticos, régimen político y ciudadanías diferenciadas. En J. Cotler y R. Cuenca (Eds.), Las desigualdades en el Perú: Balances críticos (pp. 133-168). Lima: Instituto de Estudios Peruanos.

Ríos, A. (2007). Problemas y alternativas de solución respecto a la cantidad y calidad en la formación inicial de los institutos superiores pedagógicos. Documento inédito. Lima: Proeduca-GTZ.

Rodríguez, Y. y Domínguez, R. (2009). La formación de la ciudadanía desde la experiencia escolar. Revista Peruana de Investigación Educativa, 1(1), 91-122.

Sandoval, P. (2004). Educación, ciudadanía y violencia en el Perú: una lectura del informe de la CVR. Lima: Instituto de Estudios Peruanos y Tarea.

Schulz, W., Ainley, J., Friedman, T. y Lietz, P. (2011). ICCS 2009 Latin American report. Civic knowledge and attitudes among lower-secondary students in six Latin American countries. Amsterdam: International Association for the Evaluation of Educational Achievement (IEA).

Stojnic, L. (2009). La escuela, un espacio para la democratización peruana. Un estudio comparado entre la propuesta educativa tradicional y una propuesta educativa alternativa. Revista Peruana de Investigación Educativa, 1(1), 123-162.

Stojnic, L. (2015). El efecto de la experiencia escolar en el desarrollo de actitudes favorables hacia la democracia como mejor sistema político: El caso de estudiantes peruanos recién graduados del nivel secundario. Colombia Internacional, 85, $111-139$. doi:10.7440/colombiaint85.2015.04

Stojnic, L. y Carrillo, S. (abril, 2016). Influencia de los años de educación formal y de la calidad educativa de jóvenes latinoamericanos en sus actitudes democráticas. Investigación desarrollada para la Segunda Conferencia Regional de la Iniciativa Latinoamericana para la Investigación de Políticas Públicas (ILAIPP) “Calidad e Innovación de la Educación”. Lima. 
Stojnic, L. y Román, A. (2016). Experiencia educativa universitaria y tolerancia política: entendiendo la relación desde el análisis de una muestra de estudiantes peruanos. Revista Internacional de Educación para la Justicia Social, 5(1), 139-160.

Ugarte, D. (2015). Educación Secundaria: Una deuda pendiente, un sueño que persiste. Transición del nivel primario al nivel secundario y la situación de la educación secundaria en el Perú: Problemática y oportunidades para la cooperación canadiense. Lima: Agencia Canadiense de Desarrollo Internacional (ACDI).

Yep, E. y Barea, P. (2012). Los consejos participativos regionales de educación (COPARE) y la participación de la sociedad civil. Un diagnóstico de la demandas de Ayacucho, Junín, San Martín y Ucayali. Lima: Grupo Propuesta Ciudadana.

Young, I. M. (1990). Justice and the politics of difference. Princeton, NJ: Princeton University Press.

\section{Breve CV de los autores}

\section{Ricardo Cuenca}

Doctor en educación por la Universidad Autónoma de Madrid, Magister en docencia e investigación de educación superior y psicólogo social por la Universidad Peruana Cayetano Heredia (UPCH). Sus principales áreas de interés son los temas docentes, las reformas y políticas educativas, las desigualdades sociales y la historia de la educación. Actualmente, trabaja en el Instituto de Estudios Peruanos (IEP) y lidera el equipo de investigación sobre "Trayectorias educativas, juventud y desigualdades sociales" y es profesor principal del departamento de educación de la UPCH. ORCID ID: 0000-00029459-6559. Email: rcuenca@iep.org.pe

\section{Sandra Carrillo}

Magíster en Políticas Públicas y Sociales por la Universidad Pompeu Fabra (UPF) en Barcelona y Licenciada en Psicología Educacional por la Pontificia Universidad Católica del Perú (PUCP). Sus principales áreas de interés son las políticas educativas en temas docentes, educación secundaria, educación superior, ciudadanía y gestión educativa. Actualmente, trabaja en el Instituto de Estudios Peruanos (IEP) y forma parte del equipo de investigación sobre "Trayectorias educativas, juventud y desigualdades sociales” y es docente del departamento de psicología de la PUCP. ORCID ID: O000o003-2871-4702. Email: scarrillo@iep.org.pe

\section{Luciana Reátegui}

Licenciada en sociología por la Pontificia Universidad Católica del Perú (PUCP). Su principal área de interés es la educación con énfasis en la docencia, descentralización y desigualdades socioeducativas. Actualmente, trabaja en el Instituto de Estudios Peruanos (IEP) y forma parte del equipo de investigación sobre "Trayectorias educativas, juventud y desigualdades sociales" y se desempeña como pre-docente en la especialidad de sociología de la PUCP. ORCID ID: 0000-0003-0221-2333. Email: lreategui@iep.org.pe 